\title{
Control of Pseudomonas aeruginosa blood stream infection outbreak in neonatal intensive care unit by quantitative antibiogram
}

\author{
Khyati Jain ${ }^{1}$, Madhurendra S Rajput ${ }^{2, *}$, Arjun S Gurjar ${ }^{3}$ \\ ${ }^{1,2}$ Assistant Professor, ${ }^{3}$ Tutor, Dept. of Microbiology, Amaltas Institute of Medical Sciences, Dewas, Madhya Pradesh, India
}

*Corresponding Author:

Email: dr.madhu786@gmail.com

Received: $4^{\text {th }}$ May, 2018

Accepted: $1^{\text {st June, } 2018}$

\begin{abstract}
Introduction: Neonates are very susceptible to Pseudomonas aeruginosa. Single case of Pseudomonas aeruginosa in preterm neonates is a sign for immediate action.

Aim: To describe control of an outbreak of Pseudomonas aeruginosa bloodstream infections (PA-BSI) by being vigilant and by immediate intervention, that occurred in the neonatal intensive care unit (NICU) of a teaching institution and hospital in Dewas, India.

Materials and Methods: The outbreak investigations included hunt for additional cases, evaluation of patient's records, environmental and health care worker screening, immediate reporting to authority, and detection of source by minimal available aid i.e. quantitative antibiogram.

Results: Health care worker screening samples were negative on culture for Pseudomonas aeruginosa and an environmental source was detected to be a curtain near washroom.

Conclusion: This study described control of an outbreak of PA-BSI occurring over a week among neonates which was controlled following vigorous infection control measures. Quantitative antibiogram data should be routinely used for typing purposes as an essential part of hospital associated infection control procedure in source limited settings.
\end{abstract}

Keywords: Outbreak control, Pseudomonas aeruginosa, Curtain, Quantitative antibiogram.

\section{Introduction}

Pseudomonas aeruginosa is an obligate aerobe, Gram negative rod-shaped bacterium with minimal nutritional requirements. In deficiency of wellresourced surveillance systems, reports of an outbreak control can serve to high spot serious pathogens and importance of controlling them at starting itself. Such outbreaks are regularly reported in the literature, but few of these reports come from low income countries.

Pseudomonas aeruginosa is an important cause of healthcare-associated infections, particularly among infants in neonatal intensive care units (NICUs) owing to their underdeveloped immune system and the fact that such infants are often intubated, catheterized with intravascular catheters/devices in-situ, and/or receiving parenteral nutrition, which may increase risk of infection. ${ }^{1,2}$ Predisposing factors for Pseudomonas aeruginosa infection are presence of indwelling devices, intensive care unit admission, prior antibiotic use, and length of hospital stay, underlying disease and reduced immunity. ${ }^{3,4}$ The higher survival of premature low birth-weight neonates has caused an increase in the incidence of hospital acquired $P$. aeruginosa infections. ${ }^{5}$ Although $P$. aeruginosa often only colonizes the infants, it sometimes causes infection and, despite improved treatments, $P$. aeruginosa bacteremia is fatal in $20 \%$ of cases. ${ }^{6}$ Traditional external reservoirs of $P$. aeruginosa include sinks, tubs, ventilation devices, incubators, and hand antiseptic solutions. What is more, this pathogen can also be isolated from walls, floors and even phototherapy equipment. ${ }^{7}$ There may be different transmission mechanisms, such as binding to catheters, mechanical ventilation, and the hands of medical of nurse personnel etc. ${ }^{8}$

The microbiology laboratory is at the centerpiece of attempts to watchdog the medical records of infections that can be hint to the arrival of a new microbial plague, an outbreak of any hospital associated infection. The clinical observation of two or more temporally related cases of nosocomial $P$. aeruginosa infection should raised the suspicion of an outbreak particularly in high risk pediatric patient populations (Neonatal intensive care unit, pediatric intensive care unit, oncology). ${ }^{9}$ We launched a outbreak investigation after getting two blood stream infection of Pseudomonas aeruginosa over a week. The objectives were to describe the outbreak, detect the source of the outbreak, and make recommendations for the prevention of future outbreaks. Our report highlights the importance of being vigilant, immediate implementation of infection control measures in stopping outbreak at its initial stage and use of qualitative antibiogram for surveillance of antimicrobial-resistant organisms in resource-poor settings.

\section{Materials and Methods}

Setting: AIMS, Dewas is a new setup in developing stage. The NICU consist of one room with 10 isolation division with a common attached bathroom and nursing station. Entry to NICU is supervised and basic infection control measures are followed. 
Data Collection: Neonates whose routine investigation for fever, yields growth of Pseudomonas aeruginosa on blood culture were taken into consideration. The patient folders were reviewed for the details of hospitalization and clinical history of the neonate.

Staff and Environmental Screening: A total of 76 Environmental sample were taken which includes, instrument trolley, tray, medicine trolley, weighing machine, incubator, door handle, curtain near washroom (which was wet at time of collection), sinks, tubs, swabs from taps in the patient isolation rooms, ventilation devices, water samples, water used in respiratory therapy equipment, phototherapy equipment, cleaning equipment and working station were taken. Samples from hands and apron of 18 healthcare worker posted in NICU were taken to identify the source and route of infection. 10 more neonates admitted there were also screened.

Environmental surfaces were swabbed with sterile cotton swabs, pre-moistened with sterile distilled water. Swabs were transported immediately to microbiology laboratory. Water sample $(100 \mathrm{ml})$ was collected in a commercially available sterile bottle containing sodium thiosulphate powder.

Consent from health care workers was not taken as screening of staff for possible organism carriage is good clinical practice and mandatory for public health intervention in such type of outbreak investigation.

Microbiological Testing: Microbiology testing was done in AIMS microbiology laboratory. Screening specimens were plated onto nutrient agar, blood agar and MacConkey agar. Pseudomonas aeruginosa was identified as Gram negative bacilli, motile, oxidase positive and production of green pigment. Kirby bauer disc diffusion method was done for Antimicrobial susceptibility determination, which were then interpreted according to Clinical Laboratory Standards Institute (CLSI) guidelines. ${ }^{10}$ The following antibiotics were tested: amikacin, aztreonam, ceftriaxone, ceftazidime, piperacillin tazobactam, meropenem, ciprofloxacin, norfloxacin and gentamicin.

Water samples were processed by membrane filtration, $24 \mathrm{hrs}$ incubation of the membrane filter in thioglycolate broth, subculture and subsequent identification. ${ }^{11}$

\section{Results}

Description of Outbreak and Data Collection: In current outbreak, two consecutive bloodstream infection of Pseudomonas aeruginosa showing similar quantitative antibiogram in neonate over a week period 3-6-16 to 8-6-16, raised suspicion of outbreak in NICU. Case 1: A term baby boy three days old, born to a primigravida with no adverse antenatal events was admitted to NICU with probable diagnosis of meconium aspiration syndrome. Laboratory results showed that total WBC count increased and elevated Creactive protein. Blood culture grew Pseudomonas aeruginosa. Child was treated with Amikacin and Cefotaxime. There was clinical improvement and was discharged after complete antibiotic course.

Case 2: A term female baby two day old, with respiratory distress was admitted in NICU. Her WBC count was highly raised. Blood culture grew Pseudomonas aeruginosa. Child was treated with Cefotaxime and Gentamicin. Child improved and discharged after complete course of antibiotics.

Microbiological Testing: Both isolates produced green pigmentation and fruity smell on nutrient agar, beta hemolytic on blood agar, non lactose fermenter on MacConkey agar. They were motile, oxidase positive, urease negative and citrate positive Gram negative bacilli. Both isolates were morphologically and biochemically identical. Both isolates were having similar antibiotic sensitivity pattern i.e.

Pseudomonas aeruginosa sensitive to Amikacin (19mm ZOI), Gentamicin (22mm ZOI), Ciprofloxacin $(25 \mathrm{~mm} \mathrm{ZOI})$, norfloxacin $(20 \mathrm{~mm} \mathrm{ZOI})$, piperacillintazobactam (20mm ZOI), meropenem (18mm ZOI) and were resistant to ceftazidime (11mm ZOI), ceftriaxone $(10 \mathrm{~mm} \mathrm{ZOI})$, aztreonam $(9 \mathrm{~mm} \mathrm{ZOI})$. (ZOI- zone of inhibition)

No health care worker in NICU showed isolation of Pseudomonas aeruginosa. Pseudomonas aeruginosa with similar morphology, biochemical reaction and quantitative antibiogram was isolated in a single specimen taken from the curtain near washroom. No neonate admitted showed isolation of Pseudomonas aeruginosa.

\section{Discussion}

Now days, outbreaks of hospital associated infections due to antibiotic resistant organisms are significant infection control problem in developed as well as resource-limited countries. Middle income countries are more likely to face these outbreaks of drug resistant pathogens, where resources allow use of broad spectrum antibiotics and not adequate infection control measures and regular surveillance. Despite recognition of this multidrug resistant pathogens as a global problem, very few outbreaks are investigated and documented in low-resourced countries. ${ }^{12-14}$

Pseudomonas aeruginosa is a well known cause of invasive and non-invasive disease in the hospital setting ${ }^{15-17}$ and many studies are published linking one or more environmental samples as source. ${ }^{18-20}$ The noticeable similarity between the isolated strains in current outbreak indicates an exogenous common source. In this case, curtain near washroom was a point source of infection which was wet during collection of sample and it is well known fact that Pseudomonas aeruginosa is primarily an environmental organism that is adapted to survive in numerous conditions and is particularly well adapted to wet or damp conditions.

Multi-resistant organisms may transmit from one patient to another by direct contact or indirectly, by 
contact of healthcare workers or fomites as vectors. ${ }^{15-17}$ In current outbreak, hands of health care worker can be considered likely to be vehicle between case and source. Though screening of staff did not reveal any carrier but it may be possibility that Pseudomonas aeruginosa may be a part of transient flora of health care workers hands and eliminated by the time of screening.

The control measures taken during the current outbreak were: early detection of cases, immediate reporting to higher authority and concerned health care workers, immediate microbiological assessment as well as instigating additional contact precautions for all cases (strict hand hygiene, correct use of gloves and gown) and cohorting of cases. ${ }^{21-23}$ After identification of source, the curtain was removed and health care workers were reeducated and instructed about health care practices.

The infection control procedures in place should be able to contain the infection of sporadically introduced Pseudomonas aeruginosa into NICU. In current outbreak, early identification and immediate decontamination of source, had lead to stoppage of transmission of infection in the first place. This indicates the significance of consistent and continuous surveillance of hospital associated infection among high risk patients. In low resource settings, surveillance of such pathogens should also incorporate molecular methods to support epidemiological data.

Passive surveillance is the strategy where problems are detected by using data obtained in the routine management of patient. Monitoring the records of laboratories allows early detection and response for mandatory changes in infection control procedures as well as implementation of additional surveillance methods to cut off new health care-associated infections at an initial stage. In light of this study, high-quality diagnostic microbiology laboratories are mandatory to support the infrastructure for help of the health care providers in management and control of health care associated infections. Modern hospital epidemiology began in the mid- $1960 \mathrm{~s}^{24}$ and from that time the clinical microbiology laboratory has played the critical roles it can serve for management and control of health care associated infectious diseases.

In general, the current outbreak studied, provide evidence that Quantitative antibiogram typing using inhibition zone diameter is a simple, rapid, readily available method. It appears to be suitable for prospective surveillance in a hospital which does not have molecular typing facilities.

\section{Conclusion}

12. Pitout JD, Revathi G, Chow BL, Kabera B, Kariuki S. Metallobeta-lactamase-producing Pseudomonas aeruginosa isolated from a large tertiary in Kenya. Clin Microbiol Infect. 2008;14:755-759.

13. Hammami S, Boutiba-Ben Boubaker I, Ghozzi R, Saidani $\mathrm{M}$, Amine S. Nosocomial outbreak of imipenem-resistant
This report highlights the problem of hospital associated infections and infection control challenges that they pose in a resource limited setting. It also emphasizes strict passive surveillance and use of quantitative antibiogram as a tool for microbiology laboratory to identify outbreak and its source in a low resourced and simple setup laboratory.

\section{Acknowledgment}

The authors like to address Prof. Vilas Jahagirdar, bacteriology technician incharge Miss Rinku Parindiyal, bacteriology technician Mrs. Trupti Bhopale, concerned NICU faculties and our dean of AIMS, Dewas.

\section{References}

1. Jarvis, W. R., Olson, D., Tablan, O. \& Martone, W. J. The epidemiology of nosocomial Pseudomonas cepacia infections: endemic infections. Eur J Epidemiol. 1987;3(3):233-236.

2. Gaynes, R., Edwards, J. R. \& National Nosocomial Infections Surveillance System. Overview of nosocomial infections caused by Gram-negative bacilli. Clin Infect Dis. 2005;41:848-854.

3. Harris AD, Perencevich E, Roghmann MC, Morris G, Kaye KS. Risk factors for piperacillin-tazobactamresistant Pseudomonas aeruginosa among hospitalized patients. Antimicrob Agents Chemother. 2002;46:854858.

4. Joo EJ, Kang CI, Ha YE, Kim J, Kang SJ. Clinical predictors of Pseudomonas aeruginosa bacteremia among Gram-negative bacterial infections in non-neutropenic patients with solid tumor. J Infect. 2011;63:207-214.

5. Zafar N, Wallace CM, Kieffer P, Schroeder P, Schootman $M$. Improving survival of vulnerable infants increases neonatal intensive care unit nosocomial infection rate. Arch Pediatr Adolesc Med. 2001;155:1098-1104.

6. Kuikka A, Valtonen VV. Factors associated with improved outcome of Pseudomonas aeruginosa bacteremia in a Finnish university hospital. Eur J Clin Microbiol Infect Dis. 1998;17:701-708.

7. Foca MD. Pseudomonas aeruginosa infections in the neonatal intensive care unit. Semin Perinatol. 2002;26:332-339.

8. Thuong M, Arvaniti K, Ruimy R, de la Salmonière P, Scanvic-Hameg A. Epidemiology of Pseudomonas aeruginosa and risk factors for carriage acquisition in an intensive care unit. J Hosp Infect. 2003;53:274-282.

9. Srinivasan A, Linda L. Wolfenden, Xiaoyan Song, Karen Mackie. An outbreak of Pseudomonas aeruginosa infections associated with flexible bronchoscopes. $\mathrm{N} \mathrm{Egl}$ J Med. 2003;348:221-227.

10. Clinical and Laboratory Standards Institute (2010) Performance standards for antimicrobial susceptibility testing; twentieth informational supplement. Wayne, Pennsylvania.

11. National Health Laboratory Service Standard Operating Procedure MIC1605v1 (2011) Testing for pathogens in water by membrane filtration method.

Pseudomonas aeruginosa producing VIM-2 metallo-betalactamase in a kidney transplantation unit. Diagn Pathol. 2011;6:106.

14. Crespo MP, Woodford N, Sinclair A, Kaufmann ME, Turton J. Outbreak of carbapenem-resistant Pseudomonas aeruginosa producing VIM-8, a novel metallo-beta- 
lactamase, in a tertiary care center in Cali, Colombia. $J$ Clin Microbiol. 2004;42:5094-5101.

15. Mayank D, Anshuman M, Singh RK, Afzal A, Baronia AK. (2009) Nosocomial cross-transmission of Pseudomonas aeruginosa between patients in a tertiary intensive care unit. Indian J Pathol Microbiol. 2009;52:509-513.

16. Kominos SD, Copeland CE, Grosiak B. Mode of transmission of Pseudomonas aeruginosa in a burn unit and an intensive care unit in a general hospital. Appl Microbiol. 1972;23:309-312.

17. Widmer AF, Wenzel RP, Trilla A, Bale MJ, Jones RN. Outbreak of Pseudomonas aeruginosa infections in a surgical intensive care unit: probable transmission via hands of a health care worker. Clin Infect Dis. 1993;16:372-376.

18. Majumdar, S., Kirby, A., Berry, N., Williams, C., Hassan, I., Eddleston, J. \& Burnie, J. P. An outbreak of imipenem-resistant Pseudomonas aeruginosa in an intensive care unit. J Hosp Infect. 2004;58:160-161.

19. Sa' nchez-Carrillo, C., Padilla, B., Marin, M., Rivera, M., Cercenado, E., Vigil, D., Sa' nchez-Luna, M. \& Bouza, E. Contaminated feeding bottles: the source of an outbreak of Pseudomonas aeruginosa infections in a neonatal intensive care unit. American Journal of Infection Control. 2009;37:150-154.

20. Naze, F., Jouen, E., Randriamahazo, R. T., Simac, C., Laurent, P., Ble' riot, A., et al. A. Pseudomonas aeruginosa outbreak linked to mineral water bottles in a neonatal intensive care unit: fast typing by use of highresolution melting analysis of a variable-number tandem-repeat locus. J Clin Microbiol. 2010;48:31463152.

21. Pessoa-Silva CL, Hugonnet S, Pfister R, Touveneau S, Dharan S. Reduction of health care associated infection risk in neonates by successful hand hygiene promotion. Pediatrics. 2007;120:e382-390.

22. Herruzo-Cabrera R, Garcia-Caballero J, Martin-Moreno JM, Graciani-Perez- Regadera MA, Perez-Rodriguez J. Clinical assay of N-duopropenide alcohol solution on hand application in newborn and pediatric intensive care units: control of an outbreak of multiresistant Klebsiella pneumoniae in a newborn intensive care unit with this measure. Am J Infect Control. 2001;29:162-167.

23. Won SP, Chou HC, Hsieh WS, Chen CY, Huang SM. Handwashing program for the prevention of nosocomial infections in a neonatal intensive care unit. Infect Control Hosp Epidemiol. 2004;25:742-746.

24. LaForce, F. M. 1997. The control of infections in hospitals: 1750-1950, p.1-32. In R. P. Wenzel, Prevention and control of nosocomial infections, $3^{\text {rd }}$ ed. Williams \& Wilkins, Baltimore, Md.

How to cite this article: Jain K, Rajput MS, Gurjar AS. Control of Pseudomonas aeruginosa blood stream infection outbreak in neonatal intensive care unit by quantitative antibiogram. Indian J Microbiol Res. 2018;5(3):378-381. 